\title{
Managing climate change risks is imperative for human health
}

\author{
Kristie L. Ebi
}

In 2021, extreme weather and climate events caused preventable injuries, illnesses and deaths. A clear imperative exists to reduce greenhouse gas emissions and increase the sustainability and climate resilience of health systems. Countries and communities must implement strategies to mitigate climate change and invest in health systems to protect their populations.

In 2021, climate change fuelled heatwaves, cold spells, forest fires and flooding, causing suffering and avoidable deaths worldwide. The heat dome that developed over the US Pacific Northwest and western Canada at the end of June was so intense that the occurrence of such an event would be almost impossible in the absence of human-caused climate change ${ }^{1}$. The heatwave caused more than 1,000 excess deaths and a 69-fold increase in emergency department visits, compared with the same period in 2019. Critical infrastructure was affected and high intertidal temperatures combined with low tides resulted in the death of over a million shellfish, affecting livelihoods, food security and recreation ${ }^{2}$. In July, heavy rainfall caused severe flooding that resulted in at least 184 deaths in Germany and 38 in Belgium, as well as considerable infrastructure damage ${ }^{3}$. The likelihood of this extreme rainfall event was increased by climate change. Other extreme events in 2021 included wildfires in Algeria, California, Greece and Turkey; and flooding in Australia, Japan and China. No inhabited continent was unaffected. Similar to the COVID-19 pandemic, these extreme events affected all of society and highlighted particularly vulnerable populations and regions as well as the wide range of interconnections within and across communities.

Observations of climate change in our own back gardens were reinforced by the Working Group I contribution to the $6^{\text {th }}$ Assessment Report of the Intergovernmental Panel on Climate Change, which concluded that recent changes in the climate are widespread, rapid

recent changes in the climate are widespread, rapid, intensifying and unprecedented

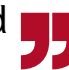

and intensifying, and unprecedented in millennia ${ }^{4}$. Heat extremes over land that were once-in-50-year events between 1850 and 1900 now occur 4.8 times more often. Such events are projected to occur 8.6 times more often with global warming of $1.5^{\circ} \mathrm{C}$, and 13.9 times more often with warming of $2{ }^{\circ} \mathrm{C}$.

Changes in the mean and variability of weather variables, and in sea levels, matter for health. A 2021 study reported that record temperatures in 2020 resulted in 3.1 billion more person-days of heatwave exposure among adults aged $\geq 65$ years and 626 million more person-days among children aged $<1$ year compared with the 1986-2005 average ${ }^{5}$. During 1991-2018, 37.0\% (range 20.5-76.3\%) of warm-season heat-related deaths across 732 locations in 43 countries were attributed to anthropogenic climate change ${ }^{6}$. Mortality varied geographically, ranging from dozens to hundreds of deaths annually, most of which were potentially preventable with effective heat action plans. Current low levels of preparedness for extreme weather events mean that increased investment in research and implementation could save lives in the current climate and in a warmer future.

Heat-related mortality is a subset of the health outcomes of concern in a changing climate. The Lancet Countdown is an international collaboration that independently monitors the health consequences of climate change ${ }^{5}$. Their 2021 report is subtitled 'code red for a healthy future' and covers trends in 44 indicators, providing a clear imperative for increasing efforts to reduce greenhouse gas emissions and thereby slow climate change, and for increasing sustainability and resilience by prioritizing population and planetary health. The report concludes that climate change is beginning to reverse years of progress in reducing water and food insecurity. A record high proportion of the land surface (19\%) was affected by extreme drought in 2020 , reducing yields of major staple crops, climate change is beginning to reverse years of progress in reducing water and food insecurity

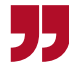

including maize $(-6 \%)$, winter wheat $(-3 \%)$, soybean $(-5.4 \%)$ and rice $(-18 \%)$, compared with 1981-2010.

The Lancet Countdown report also found that rising temperatures are increasing the geographical area that is suitable for transmission of a wide range of waterborne, foodborne and vector-borne pathogens ${ }^{5}$. Although socioeconomic development, investments in health systems and medical advances have reduced transmission of these pathogens, particularly in high- and high-middle income countries, the populations of low-income countries or regions and marginalized and/or under-resourced communities are particularly at risk.

A 2021 analysis used an evidence-based approach to estimate the impact of climatechange-induced temperature increases on the risks of heat-related morbidity and mortality, ozone-related mortality, malaria, Lyme disease, West Nile fever and diseases carried by various species of Aedes mosquito, such as dengue fever and Zika virus ${ }^{7}$. The assessment determined the temperatures at which risks increase from undetectable to medium, high and very high levels compared to the pre-industrial baseline, under three scenarios of future development. Recent climate change has probably already increased risks to moderate for heat-related morbidity and mortality, ozone-related mortality, dengue, Lyme disease and West Nile fever (FIG. 1). A detectable

\section{Key advances}

- Climate change is already causing preventable injuries, illnesses and deaths ${ }^{5}$; the health burden is projected to increase with each additional unit of warming?

- Our short-term future will be characterized by increasingly frequent and intense extreme weather and climate events, for which communities are ill-prepared ${ }^{5}$.

- Indicators of the impacts of and responses to climate change provide a clear imperative for reducing greenhouse gas emissions and increasing the sustainability and climate resilience of health systems ${ }^{5}$.

- Current investments in research and building climate-resilient health systems are insufficient to effectively manage increasing health risks ${ }^{5}$.

- The economic value of the health benefits of policies to mitigate climate change exceeds the estimated costs of mitigation ${ }^{8}$. 
impact of climate change on malaria is not yet apparent but is expected with additional warming. The risks for each health outcome are projected to increase with each increase in global mean surface temperature, with higher levels of health system adaptation moderating the extent of risks.

These increasing health risks are not being matched by investments in research and strengthening of health systems. In 2020, $63 \%$ of countries did not have a high level of implementation of national emergency frameworks ${ }^{5}$. Among 91 countries that completed a World Health Organization survey on climate change and health, only 47 reported a national adaptation plan, with insufficient resources being the main barrier to progress. Only $0.3 \%$ of US $\$ 5.1$ billion of global climate change adaptation funding was directed at health systems between 2018 and 2020, leaving vulnerable populations and regions without the critical human and financial resources

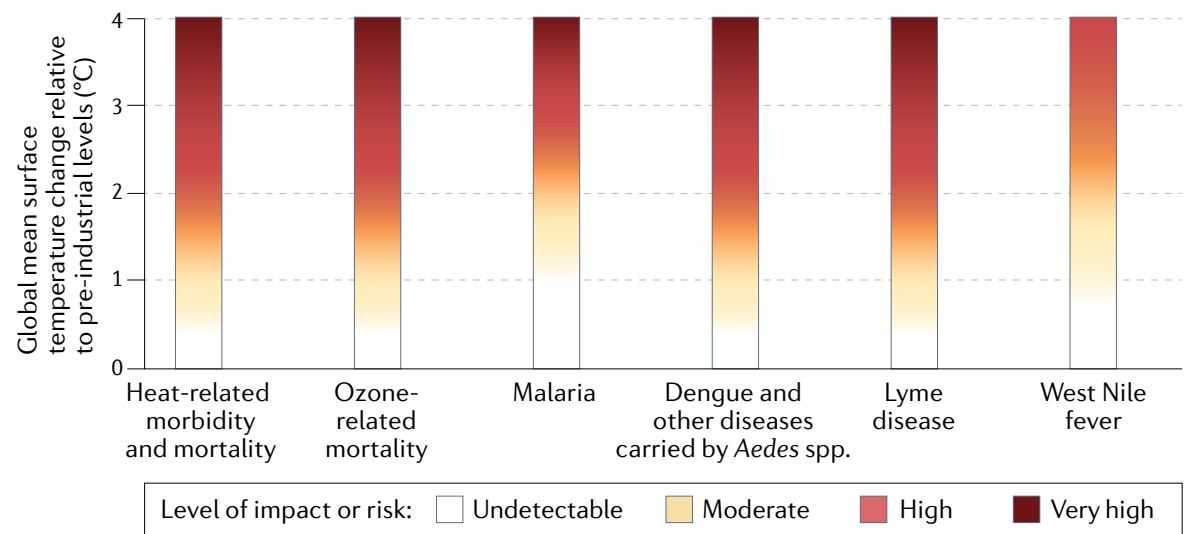

Fig. 1 | Burning embers for health outcomes. Observed and projected changes in health risks as global mean surface temperature increases above preindustrial levels, under a scenario in which the world has moderate challenges to adaptation and mitigation. The levels of impact or risk are defined as undetectable (no impacts are detectable and attributable to climate change), moderate (impacts and/or risks are detectable and attributable to climate change with at least medium confidence), high (severe and widespread impacts and/or risks) and very high (very high risks of severe impacts and/or risks and the presence of substantial irreversibility or the persistence of climate-related hazards, combined with limited ability to adapt owing to the nature of the hazard or impacts and/or risks). Adapted from REF. ${ }^{7}$, CC BY 4.0 (https://creativecommons.org/licenses/by/4.0/).
Climate Change', held in Glasgow, Scotland, in November 2021, brought the health risks of climate change to the fore, with countries committing to developing low-carbon, sustainable health systems ${ }^{10}$. There is much to celebrate in this agreement, while acknowledging the need to further increase the level of ambition to both decarbonize our economies and to increase investment in adaptation for the communities and regions at highest risk. Our future is in our hands.

Kristie L. Ebi iD

Center for Health and the Global Environment, University of Washington, Seattle, WA, USA.

e-mail:krisebi@uw.edu

https://doi.org/10.1038/s41581-021-00523-2

1. Philip, S. Y. et al. Rapid attribution analysis of the extraordinary heatwave on the Pacific Coast of the US and Canada June 2021. Earth Syst Dyn. https://doi.org/10.5194/esd-2021-90 (2021).

2. Washington Sea Grant and the Washington Department of Fish and Wildlife. Impacts from the summer 2021 heatwave on Washington shellfish. WSG News Blog, https://go.nature.com/2ZofAy7 (21 July 2021).

3. World Weather Attribution. Heavy rainfall which led to severe flooding in Western Europe made more likely by climate change. worldweatherattribution.org, https://go.nature.com/318tyvC (23 August 2021).

4. IPCC. in Climate Change 2021: The Physical Science Basis. Contribution of Working Group I to the Sixth Assessment Report of the Intergovernmental Panel on Climate Change (eds. Masson-Delmotte, V. et al.) 1-41 (IPCC, 2021).

5. Romanello, M. et al. The 2021 report of the Lancet Countdown on health and climate change: code red for a healthy future. Lancet 398 , 1619-1662 (2021).

6. Vicedo-Cabrera, A. M. et al. The burden of heat-related mortality attributable to recent human-induced climate change. Nat. Clim. Chang. 11, 492-500 (2021).

7. Ebi, K. L. et al. Burning embers: synthesis of the health risks of climate change. Environ. Res. Lett. 16, 044042 (2021).

8. Martin, M. A. et al. Ten new insights in climate science 2021: a horizon scan. Glob. Sustain. 4 E25 (2021).

9. Hess, J. J. et al. Guidelines for modeling and reporting health effects of climate change mitigation actions. Environ. Health Perspect. 128, 115001 (2020).

10. World Health Organization. Countries commit to develop climate-smart health care at COP26 UN climate conference. who.int, https://go.nature. com/3E6TCPa (9 November 2021).

Competing interests

The author declares no competing interests. 